\title{
Design of the adaptive log-domain low-pass filter
}

\author{
Sheng Qian Ma ${ }^{1, a}$, Li Rong Zheng ${ }^{1}$, Yong Jie $\mathrm{Ma}^{1}$,Wei Zhao Zhang ${ }^{1}$ and Zhi Feng $\mathrm{Ma}^{2}$ \\ ${ }^{1}$ College of Physics and Electronic Engineering, Northwest Normal University, Lanzhou, China \\ ${ }^{2}$ College of Zhixing, Northwest Normal University, Lanzhou, China
}

\begin{abstract}
This paper presents the structure and realization method of the current-controlled adaptive low-pass filter which based on the design of LC ladder network. After amplifying, amplitude limiting and shaping, the input voltage signal was converted into frequency signal. Then by the broadband frequency-current conversion circuit it was converted into current signal which has a linear relationship with input frequency. The current signal was as the bias current to adjust the cut-off frequency of the log-domain low-pass filter. So that frequency of the filter can automatically track the input signal frequency. In this paper, the design principle was introduced in detail, the design formulas were derived and the circuit of adaptive second order log-domain low-pass filter was given. The filter can realize the scope of the cut-off frequency up to $30 \mathrm{MHz}$. The tracking range for the input frequency is from $1 \mathrm{MHz}$ to $30 \mathrm{MHz}$. The simulation results show the effectiveness of design method.
\end{abstract}

\section{Introduction}

Filter has a wide range of applications in the fields of the signal processing, the data transmission and interference suppression [1-2]. Especially, adaptive filter plays an important role in the field of the adaptive signal processing and has the distinctive features of filter frequency following the change of input signal frequency automatically [3-4]. When signal processing, the test signal will inevitably be doped with noise. The filter which has fixed frequency can only realize signal filtering within a small frequency range. However, the adaptive filter can change the filter frequency according to the change of the signal, it is good to suppress the noise, and it has good filtering performance.

At present, there are many kinds of methods to implement analog adaptive filter. The first is the state variable method which can realize low-pass, high-pass, band-pass, band-stop and all-pass filter simultaneously, but the design and debug are troublesome and difficult to control, and besides the components are discrete and the non-linear effects are large [5-7]. The second method is the voltage-controlled method. Using this method, the offset voltage and drift will directly affect the stability of the low frequency [8-9]. The third method is using existing integrated filter chip. This method does not require to know the signal frequency in advance, the stability is better and cut-off or central frequency is controllable, but there are some large circuit noise and signal aliasing problems in the course of the use, even through clock frequency or pin programming is difficult to achieve the filter cut-off frequency or central frequency continuous change [10-12]. If the signal

\footnotetext{
${ }^{a}$ Corresponding author: s.q.ma@163.com
}

frequency changes in a wide range, the above three tracking methods are unable to achieve the fast tracking of the signal frequency. Log-domain filter is a current mode circuit in the true sense, which has been widely used in electronic systems by virtue of the advantages of power supply voltage, low power consumption, wide dynamic range, high frequency, wide tuning range and good linearity, and improves signal processing accuracy further [13-14]. This paper presents a design method of analog adaptive filter which use high-performance broadband frequency current converter $(\mathrm{F} / \mathrm{I})$ circuit, combined with the log-domain filter with transistors as the core elements to achieve the analog filter whose cutoff frequency can fast adapt the change of processed signal frequency automatically. The design of log-domain adaptive filter compared to other adaptive filters, it has the advantages of a low power consumption, high filtering frequency, wide dynamic range and low distortion. It can be applied in these cases: a wide range of signal frequency changing, a high-speed of the filtering, and filter frequency is not fixed.

\section{Working principle}

The principle diagram of the adaptive log-domain lowpass filter is shown in figure 1 . The input voltage signal $V_{\mathrm{i}}$ gets through the amplifier to output voltage signal $V_{\mathrm{x}}$.Then the voltage signal $V_{\mathrm{x}}$ is divided into two paths, the first will get through the limiting circuit and overvoltage comparison circuit which MAX903 is the core component to be shaped to send signal into the broadband $\mathrm{F} / \mathrm{I}$ circuit, and then the bias current signal $I_{\mathrm{B}}$ output from 
F/I circuit is sent to the log-domain low-pass filter to control the cut-off frequency. The other one will get through the voltage current conversion (V/I) circuit to send current signal $i_{\mathrm{i}}$ into the logarithmic compression circuit to be pre-processed, and then the logarithmic compression circuit output current signal $i$ is as the input signal of the log-domain low-pass filter.

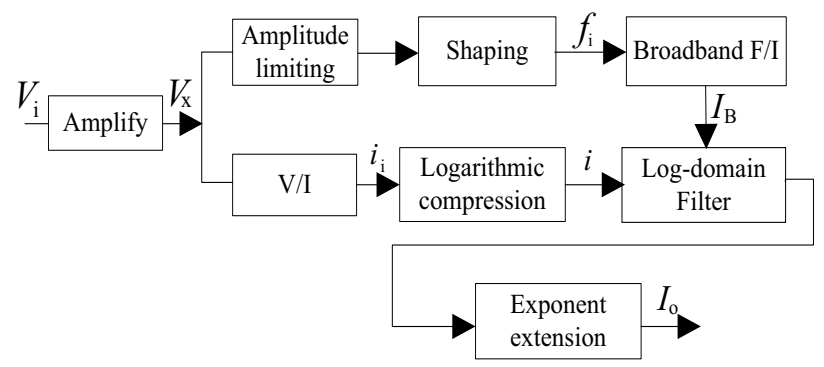

Figure 1. The diagram of adaptive log-domain low-pass filter.

\subsection{Broadband frequency current conversion(F/I) circuit}

The principle diagram of the broadband F/I circuit is shown in figure 2 , which consists of the main control circuit, the frequency division circuit, the F/I circuit and the current amplification circuit. Since the chip AD650 which in the F/I circuit maximum switching frequency is $1 \mathrm{MHz}$, so the high frequency signal to be processed first need dividing frequency after shaping. The principle of realizing broadband $\mathrm{F} / \mathrm{I}$ circuit is the processed frequency signal $f_{\mathrm{i}}$ after shaping first gets through 400 frequency division circuit to send signal $f_{\mathrm{i} 1}$ into main control circuit in which control chip is 89s52. Main control circuit calculates and compares parameters in its internal to choose the appropriate frequency division multiple, and then controls frequency division circuit to re-divide frequency for the signal after shaping. Meanwhile, it controls current amplification circuit to generate the corresponding magnification. The re-divided frequency signal gets through F/I circuit to convert frequency signal to current signal, and it will go into current amplification circuit. The $I_{\mathrm{B}}$ is output current of broadband F/I circuit.

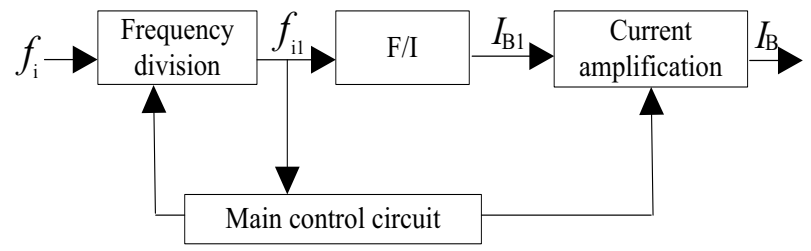

Figure 2. The diagram of broadband frequency-current conversion circuit.

The F/I of figure 2 is shown in figure 3, it converts input pulse signal into current signal. Input pulse frequency has a linear relationship with output current value, so it is possible to measure the input pulse frequency indirectly by measuring the output current value. F/I circuit is composed of the frequency voltage (F/V) conversion chip AD650, operational amplifier OP37 and a small amount of resistors and capacitors.
In figure 3 , the input pulse $f_{\mathrm{i} 1}$ through the coupling capacitor $C_{1}$ to be sent into AD650 internal comparator, when its potential is low to the comparator reference potential $(-0.6 \mathrm{~V})$, internal current switch to end of integral capacitor $C_{\mathrm{INT}}$ and charge for it. When the leakage current on $R_{4}$ and the charging current on $C_{\mathrm{INT}}$ is equal, the voltage on the $C_{\mathrm{INT}}$ is stability. The output voltage $U_{\mathrm{i}}$ of pin 1 is proportional to the input frequency $f_{\text {il }}$, that is

$$
U_{\mathrm{i}}=t_{\mathrm{OS}} \times R_{\mathrm{INT}} \times f_{\mathrm{i} 1}=\left(C_{\mathrm{OS}} \times 6.8 \times 10^{3}+3.0 \times 10^{-7}\right) \times R_{4} \times f_{\mathrm{i} 1}
$$

Then input $U_{\mathrm{i}}$ into the subsequent $\mathrm{V} / \mathrm{I}$ circuit in which operational amplifier OP37 is as the core component.

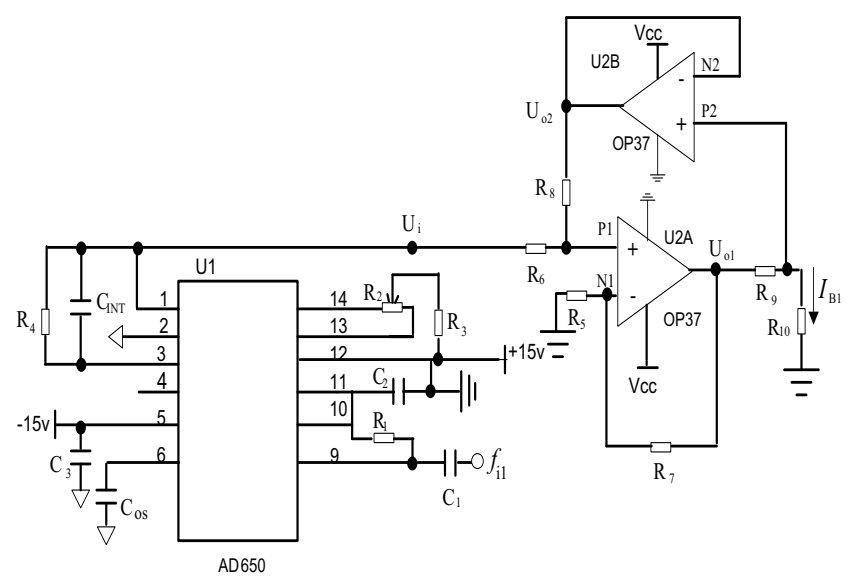

Figure 3. F/I circuit.

For the op-amp U2A and U2B, it can obtain

$$
\begin{aligned}
& U_{\mathrm{O} 1}=\left(R_{7} / R_{5}+1\right) U_{\mathrm{P} 1} \\
& U_{\mathrm{P} 1}=U_{\mathrm{i}} R_{8} /\left(R_{6}+R_{8}\right)+U_{\mathrm{P} 2} R_{6} /\left(R_{6}+R_{8}\right)
\end{aligned}
$$

The voltage on $R_{9}$ is $U_{\mathrm{R} 9}=U_{\mathrm{o} 1}-U_{\mathrm{P} 2}$, the eqution (2) and (3) are substituted into $U_{\mathrm{R} 9}$, it can deduce

$$
U_{R_{9}}=\left(1+R_{7} / R_{5}\right)\left[U_{\mathrm{i}} R_{8} /\left(R_{6}+R_{8}\right)+U_{\mathrm{P} 2} R_{6} /\left(R_{6}+R_{8}\right)\right]-U_{\mathrm{P} 2}
$$

When taking $R_{5}=R_{6}=R_{7}=R_{8}=47 \mathrm{~K}$, then $U_{\mathrm{R} 9}=U_{\mathrm{i}}$, it can deduce

$$
I_{\mathrm{B} 1}=U_{R_{9}} / R_{9}=U_{\mathrm{i}} / R_{9}
$$

The eqution (1) is substituted into eqution (5), and due to $I_{\mathrm{B}}=m I_{\mathrm{B} 1}, f_{\mathrm{i}}=m f_{\mathrm{i} 1}$, in figure $2, m=400$. it can deduce

$$
I_{\mathrm{B}}=\left[\left(C_{\mathrm{OS}} \times 6.8 \times 10^{3}+3.0 \times 10^{-7}\right) \times R_{4} / R_{9}\right] \times f_{\mathrm{i}}
$$

It can be seen from eqution (6) the output current will change linearly with input frequency as long as adjusting the value of resistors and capacitors reasonably.

\subsection{Log-domain filter}

There is a strict logarithmic relationship between the input voltage and output current of the transistor $v_{\mathrm{BE}}=V_{\mathrm{T}}$ $\ln \left(I_{\mathrm{C}} / I_{\mathrm{S}}\right)$. According to the characteristics, people use 
transistors as core component to build the log-domain filter circuit, so as to realize linear circuits by using nonlinear components. Compared with the traditional filter, log-domain filter has advantages in the frequency characteristic, processing speed, and signal bandwidth. It can meet the requirements of wide tuning range and good linearity when the modern electronic products work under the condition of low voltage.

Log-domain filter is composed of logarithmic compression circuit, the state space of nonlinear filter circuit and the exponent extension circuit, its composition block diagram is shown in figure 4 . Input current signal gets through the logarithmic compression circuit to be processed and converted into a logarithmic domain. Weak signals are amplified to a much greater than the noise signal level, and strong signals are compressed to less than distortion level, thus preventing signal distortion and expanding the dynamic range of the circuit. After logarithmic compression circuit processing, the output voltage signal is processed by the state space nonlinear filtering circuit realized by bipolar transistors, so can make the system achieve a particular filtering function. The signal after filtering is decompressed by the exponent extension circuit and converted into a linear domain. Namely process signal nonlinearly again, the weak signal is attenuated, and the strong signal is amplified, so as to restore the proportion of the original signal, making the system into a linear system.

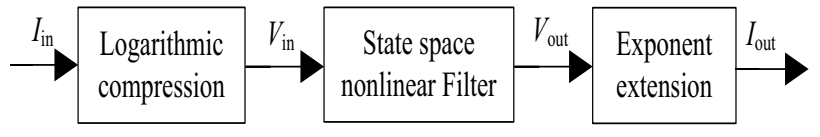

Figure 4. The diagram of log-domain filter.

\section{Design of adaptive second-order log- domain low-pass filter}

The proposed adaptive second-order log-domain lowpass filter is designed based on LC ladder network, mainly using the broadband F/I circuit, V/I circuit, the transistors, and a small amount of resistors and capacitors. The low-pass LC ladder prototype circuit is shown in figure 5 .

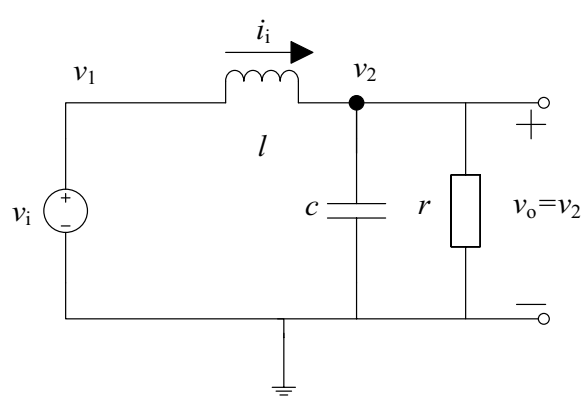

Figure 5. Prototype circuit of low-pass LC ladder.

The transfer function of the LC ladder prototype circuit is:

$$
H(\mathrm{~s})=V_{\mathrm{o}}(\mathrm{s}) / V_{\mathrm{i}}(\mathrm{s})=[1 /(l c)] /\left[\mathrm{s}^{2}+s /(r c)+1 /(l c)\right]
$$

And the transfer function of the standard low-pass filter is:

$$
H(\mathrm{~s})=K \omega_{0}^{2} /\left(s^{2}+s \omega_{0} / Q+\omega_{0}^{2}\right)
$$

Comparing the eqution (7) with (8), it can deduce cut-off frequency $\omega_{0}$ of LC ladder filter:

$$
\omega_{0}=1 / \sqrt{l c}
$$

quality factor $Q=r \sqrt{c / l}$ and dc gain $K=1$.

The log-domain filter corresponding to the LC ladder filter is shown in figure 6 , it is made up of transistors and a small amount of capacitors.

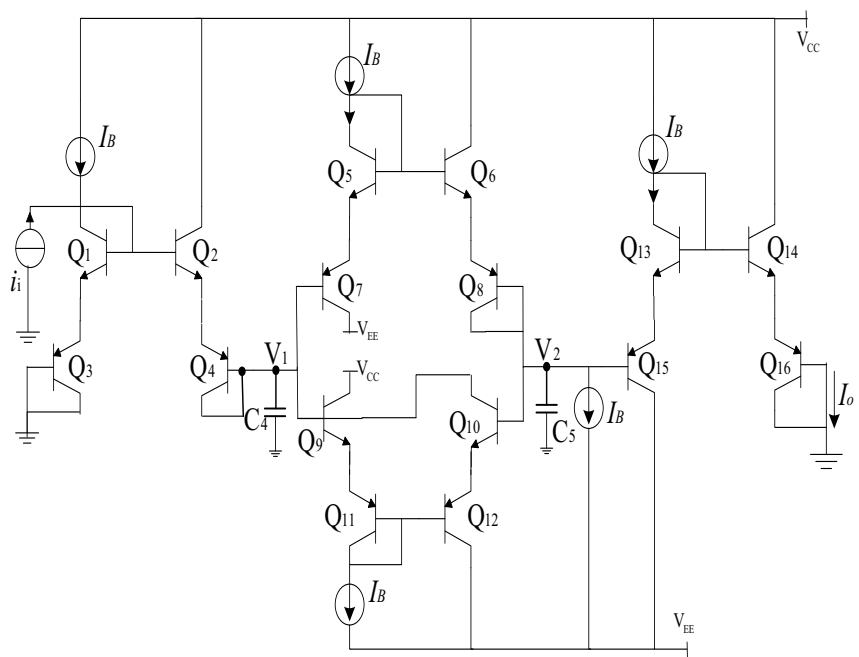

Figure 6. The circuit of log-domain filter.

The in-phase integrator which is composed by transistors Q1-Q4 and capacitor $C_{4}$ implement in-phase integral operation and logarithmic operation for the input current $i_{\mathrm{i}}$. Transistors Q9-Q12 and capacitor $C_{5}$ realize inverse integral operation to voltage $V_{2}$. Transistors Q13Q16 controlled by the bias current compose exponent extension circuit. $I_{\mathrm{B}}$ is produced by figure 2 , which is as the bias current control end of the log-domain filter.

According to Kirchhoff's law for capacitor $C_{4}$ node, we can obtain:

$$
C_{4} d V_{1} / d t=i_{\mathrm{i}} e^{-V_{1} / 2 V_{\mathrm{T}}}+I_{\mathrm{B}} e^{-V_{1} / 2 V_{\mathrm{T}}}-I_{\mathrm{B}} e^{\left(V_{2}-V_{1}\right) / 2 V_{\mathrm{T}}}
$$

On both ends of eqution (10) multiplied by $e^{V_{1} / 2 V_{\mathrm{T}}}$ at the same time and adjust the order, it can deduce

$$
2 C_{4} V_{\mathrm{T}} / I_{\mathrm{B}} \cdot\left[d / d t\left(I_{\mathrm{B}} e^{V_{\mathrm{I}} / 2 V_{\mathrm{T}}}-I_{\mathrm{B}}\right)\right]=i_{\mathrm{i}}-I_{\mathrm{B}} e^{V_{2} / 2 V_{\mathrm{T}}}+I_{\mathrm{B}}
$$

In addition, the LOG and EXP mapping function is:

$$
\operatorname{LOG}(x)=2 V_{\mathrm{T}} \ln \left[\left(I_{\mathrm{B}}+x\right) / I_{\mathrm{B}}\right] \quad \operatorname{EXP}(x)=I_{\mathrm{B}} e^{x / 2 V_{\mathrm{T}}}-I_{\mathrm{B}}
$$

The eqution (12) is substituted into eqution (11), it can deduce

$$
\operatorname{EXP}\left(V_{1}\right)=I_{\mathrm{B}} /\left(2 V_{\mathrm{T}} C_{4}\right) \cdot \int\left[i_{\mathrm{i}}-\operatorname{EXP}\left(V_{2}\right) i_{\mathrm{i}}\right] d t
$$


From figure 5, we can obtain:

$$
\operatorname{EXP}\left(V_{1}\right)=1 / l \cdot \int\left[i_{\mathrm{i}}-\operatorname{EXP}\left(V_{2}\right)\right] d t
$$

Comparing the eqution (13) with eqution (14), it can deduce

$$
l=2 V_{\mathrm{T}} C_{4} / I_{\mathrm{B}}
$$

By the same token, writing KCL equation of capacitor $C_{5}$ node and using the LOG and EXP mapping function, we can get:

$$
\operatorname{EXP}\left(V_{2}\right)=I_{\mathrm{B}} /\left(2 V_{\mathrm{T}} C_{5}\right) \cdot \int\left[\operatorname{EXP}\left(V_{1}\right)-\operatorname{EXP}\left(V_{2}\right)\right] d t
$$

From figure 5, we can obtain:

$$
\operatorname{EXP}\left(V_{2}\right)=1 / c \cdot \int\left[\operatorname{EXP}\left(V_{1}\right)-1 / r \operatorname{EXP}\left(V_{2}\right)\right] d t
$$

When taking $r=1 \Omega$, comparing the eqution (16) with eqution (17), it can deduce

$$
c=2 V_{\mathrm{T}} C_{5} / I_{\mathrm{B}}
$$

The eqution (15) and (18) are substituted into eqution (9), it can obtain

$$
\omega_{0}=I_{\mathrm{B}} /\left(2 V_{\mathrm{T}} \sqrt{C_{4} C_{5}}\right)
$$

quality factor $Q=\sqrt{C_{5} / C_{4}}$ and dc gain $K=1$.

so the cut-off frequency of log-domain filter is:

$$
f_{0}=\omega_{0} / 2 \pi=I_{\mathrm{B}} /\left(4 \pi V_{\mathrm{T}} \sqrt{C_{4} C_{5}}\right)
$$

The eqution (6) is substituted into eqution (20), it can deduce

$$
f_{0}=\left[\left(C_{\mathrm{OS}} \times 6.8 \times 10^{3}+3.0 \times 10^{-7}\right) \times R_{4}\right] /\left(4 \pi V_{\mathrm{T}} R_{9} \sqrt{C_{4} C_{5}}\right) \times f_{\mathrm{i}}
$$

It can be seen from eqution (21) that the cut-off frequency of the filter designed will change linearly with input frequency as long as adjusting the value of capacitors and resistors reasonably.

\section{Experimental result}

Design frequency range of the filter from $1 \mathrm{MHz}$ to $30 \mathrm{MHz}$. When taking $C_{\mathrm{OS}}=53 \mathrm{pf}, C_{4}=C_{5}=1010 \mathrm{pf}, R_{4}=$ $100 \Omega, R_{9}=200 \mathrm{~K} \Omega, R_{1}=2.2 \mathrm{~K} \Omega, R_{2}=20 \mathrm{~K} \Omega, R_{3}=$ $250 \mathrm{~K} \Omega, C_{1}=1000 \mathrm{pf}, C_{2}=C_{3}=0.1 \mathrm{uf}, C_{\mathrm{INT}}=3.3 \mathrm{pf}, R_{10}=$ $500 \Omega$, then according to eqution (21), it can deduce

$$
f_{0}=f_{\mathrm{i}}
$$

The above parameters are substituted into the eqution (20), it can deduce $f_{0}=3.03 \times 10^{9} I_{\mathrm{B}}$. The input current signal is taken $500 \mathrm{uA}$, the control bias current $I_{\mathrm{B}}$ are taken 330uA, 990uA, 3300uA, 6600uA, 9900uA, the simulation results with ORCAD are shown in figure 7.

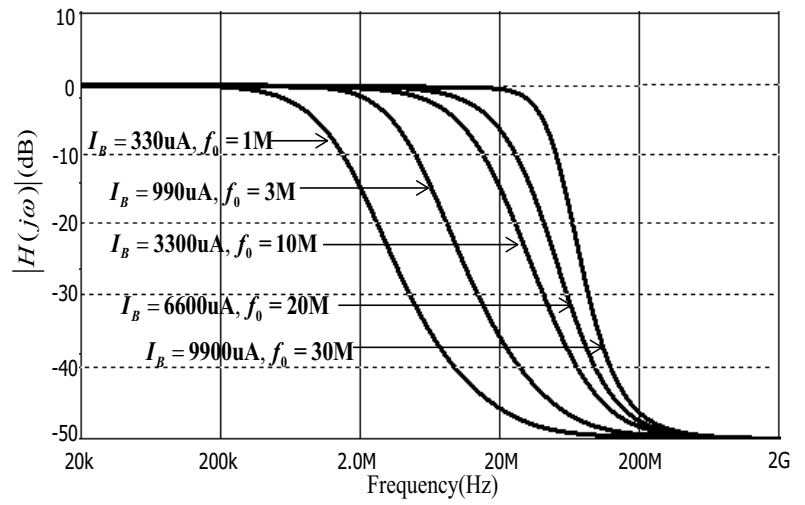

Figure 7. The amplitude-frequency responses of the log-domain second-order low-pass filter.

According to figure 7 , the analysis of second-order low-pass log-domain filter performance is shown in table 1 , including the bias current corresponding to the input frequency, cut-off frequency and stop-band attenuation rate.

Table 1. The performance of the filter.

\begin{tabular}{|c|c|c|c|}
\hline $\begin{array}{c}\text { Input } \\
\text { frequency } \\
\left(f_{\mathrm{i}} / \mathrm{MHz}\right)\end{array}$ & $\begin{array}{c}\text { Bias } \\
\text { current } \\
\left(I_{\mathrm{B}} / \mathrm{uA}\right)\end{array}$ & $\begin{array}{c}\text { Cut-off } \\
\text { frequency } \\
\left(f_{0} / \mathrm{MHz}\right)\end{array}$ & $\begin{array}{c}\text { Stop-band } \\
\text { attenuation } \\
\text { rate(dB/decade })\end{array}$ \\
\hline 1 & 330 & 1 & 40 \\
\hline 3 & 990 & 3 & 40 \\
\hline 10 & 3300 & 10 & 45 \\
\hline 20 & 6600 & 20 & 45 \\
\hline 30 & 9900 & 30 & 45 \\
\hline
\end{tabular}

Analysis the experimental results, it can obtain: the cut-off frequency $f_{0}$ of the designed second-order lowpass log-domain filter changes along with the change of the input bias current $I_{\mathrm{B}}$, and has a linear relationship with $I_{\mathrm{B}}$. When the frequency of the input signal is small, the attenuation of amplitude frequency characteristics of the filter in stop-band is not very good. When the input frequency increasing, the cut-off frequency of low-pass filter also increases accordingly, the amplitude frequency characteristics within the pass-band is relatively flat, in the stop-band attenuation rate is also increasing, the attenuation is very good. Transformation precision of the transistor is the main affecting factor. Can be seen from the overall result, the cut-off frequency of second-order low-pass log-domain filter can vary with the change of the input signal frequency, namely realizing automatic adjustment of the cut-off frequency to the input signal frequency.

\section{Conclusion}

The design method of this paper is to convert the signal frequency into current value through the broadband F/I 
circuit. The current signal, which is input into the bias current control end of the log-domain filter, controls the cut-off frequency of filter, so as to achieve automatic adaptation of the filter's frequency. The actual circuit structure is designed. When the input signal changes in frequency range from $1 \mathrm{MHz}$ to $30 \mathrm{MHz}$, the cut-off frequency of the adaptive second-order low-pass filter can automatically adapt to the change of the input signal frequency. From software simulation test of the circuit, it can see the waveform is good, and the filter amplitudefrequency characteristics within the pass-band is relatively flat, the attenuation in stop-band is good, proved the correctness of the circuit and the validity of the design. In this paper, the designed adaptive secondorder log-domain low-pass filter is mainly composed of the broadband F/I circuit, V/I circuit, transistors and a small amount of resistors and capacitors. The structure of circuit is simple and easy to adjust. And the filter system has broad filtering bandwidth, good real-time dynamic characteristics, and can be widely used in all aspects of the sensor data processing. It resolves the problem of the measured signal frequency changing in a wide range.

\section{Acknowledgement}

The authors are thankful to the Natural Science Foundation of China (61162017) and Northwest Normal University NWNU-LKQN-13-16.

\section{References}

1. D. Yang, J.Y. Wang, F. Cai. Journal of detection and control, 32(3): 79-82 (2010)

2. S.Q. Ma, X.P. Ran, M.H. Fan. Piezoelectric and light, 35(2): 245-249 (2013)

3. Rola A, Mourad N. IEEE Journal of solid-state circuits, 38(2): 205-215 (2003)

4. T.F. Wang, T.S. Wang, Z.Y. Zhang. Mechanical and electrical engineering, 32(9):1267-1270 (2015)

5. H.L. Cheng, W.Z. Ge. Semiconductor integrated circuit, 38(3): 173-188 (2013)

6. L. Cai, X.K. Ma. Journal of xi 'an jiaotong university, 37(10): 1075-1078 (2003)

7. J.J. CHU, Z.Y. Xiao, H. Li. Journal of Shanghai university (natural science edition), 20(1): 1-8 (2014)

8. L. Zhang, Y.C. Zeng, H.J. Yang. Microelectronics, 39(3): 380-384 (2009)

9. C. Kasimis, G. Souliotis, C. Psychalinos. International journal of electronics, 99(2): 197-209 (2012)

10. M. Li, Z. Wang, Y.Q. Shao. Laboratory research and exploration, 33(8): 89-92 (2014)

11. N.A. Shah, S.Z. Iqbal, N. Parveen. Analog integrated circuits and signal processing, 67(1): 85-88 (2011)

12. X.Y. Wang, H.G. Yang, T. Yin. Microelectronics and computer, 29(7): 18-22 (2012)

13. A.U. Cam. Analog integr circ sig process, 60: 221229 (2009)

14. S.M. Maria Cristina Piccirilli. Circuits syst signal process, 30: 371-389 (2011) 